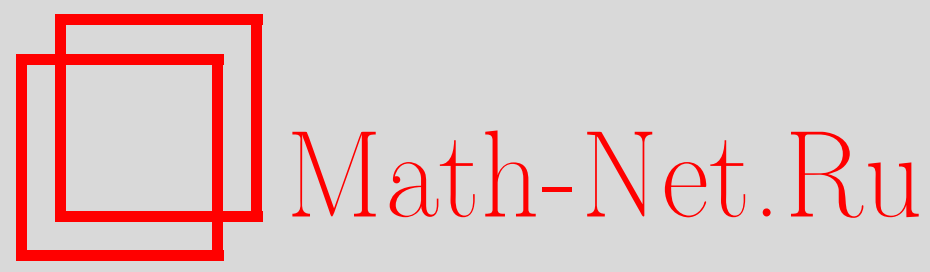

А. Б. Шаповал, Интегральное многообразие нелинейного эллиптического уравнения в цилиндре, Матем. заметки, 1997, том 61, выпуск 3, 476-480

DOI: https://doi.org/10.4213/mzm1527

Использование Общероссийского математического портала Math-Net.Ru подразумевает, что вы прочитали и согласны с пользовательским соглашением http://www . mathnet.ru/rus/agreement

Параметры загрузки:

IP: 54.237 .206 .68

26 апреля 2023 г., 05:12:49

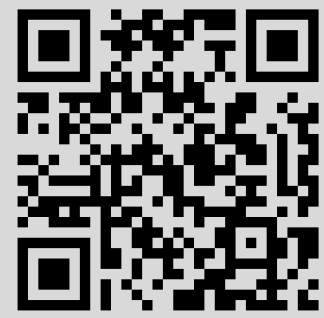




\section{ИНТЕГРАЛЬНОЕ МНОГООБРАЗИЕ НЕЛИНЕЙНОГО ЭЛЛИПТИЧЕСКОГО УРАВНЕНИЯ В ЦИЛИНДРЕ}

\section{А. Б. Шаповал}

В цилиндре $\Omega=\mathbb{R} \times \omega$, где $\omega=\left[\alpha_{1}, \alpha_{2}\right] \Subset \mathbb{R}$, рассматривается эллиптическое уравнение

$$
\partial_{t}^{2} u+2 b \partial_{t} u-A u-F(t, u)=g(t, x) .
$$

Здесь $A=-\partial_{x}^{2}, b>0$-некоторая константа, $u=u(t, x)=\left(u_{1}, \ldots, u_{m}\right), F(t, u), g(t, x)-$ векторные функции, $(t, x) \in \mathbb{R} \times \omega$. На границе ставится условие Дирихле

$$
\left.u\right|_{\partial \omega}=0 \text {. }
$$

Предполагается, что нелинейная функция $F$ удовлетворяет следуюшим условиям:

$$
\begin{gathered}
F(t, \cdot) \in C^{1}\left(\mathbb{R}^{m}\right), \quad F \in C\left(\mathbb{R} \times \mathbb{R}^{m}, \mathbb{R}^{m}\right), \\
F(t, v) . v \geqslant-C_{1}+C_{2}|v|^{2+\varepsilon}, \quad C_{1}, C_{2}, \varepsilon>0, \\
\left|\frac{\partial F(t, v)}{\partial v}\right| \leqslant M(|v|), \\
\left|F\left(t_{1}, v\right)-F\left(t_{2}, v\right)\right| \leqslant M(|v|)\left|t_{1}-t_{2}\right|,
\end{gathered}
$$

где $M: \mathbb{R}_{+} \rightarrow \mathbb{R}_{+}-$монотонная функция, а $u . v$ обозначает скалярное произведение в $\mathbb{R}^{m}$. Предположим также, что функция $g(t) \in\left(C\left(\mathbb{R}, L_{2}(\omega)\right)\right)^{m}$ имеет конечную норму

$$
|g|_{a}=\sup _{t \in \mathbb{R}}\|g(t, \cdot)\|_{\left(L_{2}(\omega)\right)^{m}}<\infty
$$

и липшицева по $t$ :

$$
\left\|g\left(t_{1}, \cdot\right)-g\left(t_{2}, \cdot\right)\right\|_{\left(L_{2}(\omega)\right)^{m} \leqslant C_{3}\left|t_{1}-t_{2}\right| .}
$$

Инерциальное многообразие уравнения (1) в автономном случае (функции $F$ и $g$ не зависят от $t$ ) построено в статье [1]. В настоящей работе доказано существование интегрального многообразия, соответствующего задаче $(1),(2)$.

Построение интегрального многообразия. Положим $H_{s}=\left(H_{s}(\omega)\right)^{m},\|\cdot\|_{s}=$ $\|\cdot\|_{H_{s}}, H=\left(L_{2}(\omega)\right)^{m},\|\cdot\|=\|\cdot\|_{H}, V_{s}\left(t_{1}, t_{2}\right)=\left(H_{s}\left(\left[t_{1}, t_{2}\right] \times \omega\right)\right)^{m}$, где $H_{s}(Q)-$ пространство Соболева порядка $s$ в области $Q$ (см. [2]). Пусть $\Omega_{\tau}=[\tau, \infty) \times \omega, \Sigma=H_{3 / 2} \cap H_{1}^{0}$. Через $\Lambda^{a}(s)$ обозначим множество функций $\varphi(t, x): \Omega_{s} \rightarrow \mathbb{R}^{m}$, принадлежаших пространствам $V_{2}(t, t+1) \forall t \geqslant s$ и имеющих конечную норму

$$
\|\varphi\|_{a, s}=\sup _{t>s}\|\varphi\|_{V_{2}(t, t+1)}
$$

Под решением задачи $(1),(2)$ в полуцилиндре $\Omega_{\tau}$ с начальным условием

$$
\left.u\right|_{t=\tau}=u_{\tau}, \quad u_{\tau} \in \Sigma,
$$

Работа выполнена при частичной поддержке Российского фонда фундаментальных исследований, грант № 96-01-00354. 
понимается функция $u \in \Lambda^{a}(\tau)$, которая удовлетворяет уравнению (1) как равенству в $H$.

Согласно [3], [4] при вьполнении перечисленных вьше условий любое решение $u$ задачи $(1),(2),(8)$ удовлетворяет неравенству

$$
\|u\|_{V_{2}(t, t+1)} \leqslant Q_{1}\left(\|u\|_{\Sigma}\right) e^{-\alpha(t-\tau)}+Q_{1}\left(|g|_{a}\right),
$$

где $\alpha>0$, а $Q_{1} \in C(\mathbb{R})$ - монотонная функция.

Определим срезающую функцию $q(\xi) \in C^{1}\left(\mathbb{R}_{+},[0,1]\right)$, обладающую следующими свойствами:

$$
\left|\frac{d q}{d \xi}\right|<2
$$

$q(s)=1$ при $0 \leqslant s \leqslant 1, q(s)=0$ при $s>2$. Положим

$$
f(t, v)=q\left(\frac{|v|^{2}}{\rho^{2}}\right) F(t, v), \quad t, v \in \mathbb{R}
$$

где $\rho=2 Q_{1}\left(|g|_{a}\right)$. Вместе с уравнением (1) рассмотрим уравнение

$$
\partial_{t}^{2} u+2 b \partial_{t} u-A u-f(t, u)=g(t, x)
$$

ЛЕмма 1. Пусть $f$ определена формулой (10), а F удовлетворяет условиям (3) $-(6)$. Тогда

$$
\begin{gathered}
|f(t, v)| \leqslant K, \quad\left|f\left(t, v_{1}\right)-f\left(t, v_{2}\right)\right| \leqslant K\left|v_{1}-v_{2}\right|, \quad t, v \in \mathbb{R} . \\
\left|f\left(t_{1}, v\right)-f\left(t_{2}, v\right)\right| \leqslant K_{1}\left|t_{1}-t_{2}\right|,
\end{gathered}
$$

Константы $K$ и $K_{1}$ зависят от числа $\rho$ и функиии $M(\xi)$ из неравенства (5).

Доказательство очевидно.

Пусть $\left\{e_{i}\right\}_{i=1}^{\infty}-$ собственные векторы оператора $A$ с граничньм условием Дирихле, соответствующие собственным значениям $\left\{\lambda_{i}\right\}$, пронумерованным в порядке возрастания. Положим $E_{+}=\operatorname{span}\left(e_{1}, \ldots, e_{N}\right), E_{-}=\left[\operatorname{span}\left(e_{N+1}, \ldots\right)\right]_{3 / 2}$, где $[B]_{3 / 2}$ обозначает замыкание множества $B$ в пространстве $H_{3 / 2}$. Обозначим через $P_{+}$ортогональный проектор из $H_{3 / 2}$ на $E_{+}, P_{-}=I-P_{+}, E=H_{3 / 2} \times E_{+}$.

Пусть $N$ - такое число, что для оператора $A$ выполнено спектральное условие

$$
\lambda_{N+1}-\lambda_{N}>l K
$$

где $K$ определено в лемме 1 , a $l \geqslant 5$. Положим $r=\sqrt{b^{2}+\lambda_{N+1}-\theta K}$. Здесь $\theta=\theta(l)-$ достаточно малое положительное число (при $l=5$ имеем $\theta=2.7$ ). Для определения интегрального многообразия введем весовые пространства $W(\tau), \bar{W}(\tau)$ функций, определенных при всех $t \in \mathbb{R}$. Нормы в этих пространствах задаются следующими формулами:

$$
\begin{aligned}
& \|u\|_{W(\tau)}=\sup _{T \in \mathbb{R}}\left[e^{-b(T-\tau)}\|u(T)\| \cosh ^{-1}(r(T-\tau))\right] \\
& \|u\|_{\bar{W}(\tau)}=\sup _{T \in \mathbb{R}}\left(\int_{T}^{T+1}\left[\|u\|_{2}^{2}+\left\|\partial_{t} u\right\|_{1}^{2}+\left\|\partial_{t}^{2} u\right\|_{0}^{2}\right] e^{2 b(t-\tau)} \cosh ^{-2}(r(t-\tau)) d t\right)^{1 / 2} .
\end{aligned}
$$


Под интегральньм многообразием уравнения (11) понимается конечномерное липшицево многообразие $M \subset \mathbb{R} \times E$, которое удовлетворяет следуюшим условиям:

(i) для любой точки $(s, p, q) \in M$ существует решение $v(t) \in \bar{W}(s)$ уравнения (11) такое, что $v(s)=p, P_{+} \partial_{t} v(s)=q$ и $\left(t, v(t), P_{+} \partial_{t} v(t)\right) \in M$ для любых $t \in \mathbb{R} ;$

(ii) для любого решения $v$ задачи $(1),(2)$ из пространства $\bar{W}(0)$ справедлива формула $\left(t, v(t), P_{+} \partial_{t} v(t)\right) \in M \forall t \in \mathbb{R}$

(iii) для любого фиксированного $s \in \mathbb{R}$ и любого решения уравнения (11) $u(t) \in \Lambda^{a}(s)$ сушествует такое решение $v(t) \in \bar{W}(s)$ уравнения (11), лежашее на $M$, что

$$
\|u(t)-v(t)\|_{\Sigma} \leqslant C_{4} e^{-\mu_{N}(t-s)}, \quad t>s .
$$

Здесь $C_{4}$ и $\mu_{N}-$ некоторые константы.

Теорема 1. Пусть выполнены условия (12), (13), (7). Тогда

1) уравнение $(11),(2)$ обладает $(2 N+1)$-мерным интегральным многообразиeм $M$;

2) для любого решения $u(t) \in \Lambda^{a}(\tau)$ уравнения $(1),(2),(8)$ существуют $t_{1} \in \mathbb{R}$ u решение $v(t) \in \Lambda^{a}\left(t_{1}\right)$ этого уравнения, лехсащее на многообразии $M$, такие, что

$$
\|u(t)-v(t)\|_{\Sigma} \leqslant C_{4} e^{-(b+r)\left(t-t_{1}\right)}, \quad t>t_{1} .
$$

Доказательство теоремы 1 основано на следующей теореме существования и единственности решения эллиптического уравнения $(11),(2),(8)$ в пространстве $\bar{W}(\tau)$.

Теорема 2. Пусть выполнены условия (12), (13). Тогда для любых $b \in \mathbb{R}, \tau \in \mathbb{R}$, $u_{+} \in E_{+}, u_{+}^{\prime} \in E_{+}, g \in W(\tau)$ существует единственное решение $u \in \bar{W}(\tau)$ уравнения (11) с начальным условием

$$
\left.P_{+} u\right|_{t=\tau}=u_{+},\left.\quad P_{+} \partial_{t} u\right|_{t=\tau}=u_{+}^{\prime}
$$

Это решение липиццево по $u_{+}, u_{+}^{\prime}$.

Доказательство проводится методами аналогичньми работе [1].

Определим отображение $\pi: \mathbb{R} \times E_{+} \times E_{+} \rightarrow E_{-}$формулой $\pi\left(\tau, u_{+}, u_{+}^{\prime}\right)=P_{-} u(\tau)$, где $u \in \bar{W}(\tau)$ - решение уравнения (11) при начальном условии (15). Согласно теореме 2 отображение $\pi$ определено корректно. Положим

$$
M=\left\{(t, u, v) \in \mathbb{R} \times H_{3 / 2} \times E_{+}: P_{-} u=\pi\left(t, P_{+} u, v\right)\right\} .
$$

\section{Экспоненциальное притяжение к многообразию $M$.}

ТЕОРема 3. Пусть выполнены условия (7), (12), (13). Пусть и(t) - решение задачи (11), (8). Тогда существует решение $v(t)$ задачи (11), (8) такое, что $(t, v(t)) \in M \quad \forall t>\tau u$

$$
\|u(t)-v(t)\|_{\Sigma} \leqslant C e^{-(b+r)(t-\tau)} \quad \forall t>\tau .
$$


ДокАЗАТЕЛЬСтво. Зафиксируем произвольные числа $T>\tau+2$ и $l>T+1$. Пусть $\psi(t ; \tau, l)=0$ при $t \leqslant \tau$ и $t \geqslant l ; \psi(t ; \tau, l)=1$ при $\tau+1 \leqslant t \leqslant l-1,\left|\psi_{t}^{\prime}(t ; \tau, l)\right| \leqslant 2$, $\left|\psi_{t}^{\prime \prime}(t ; \tau, l)\right| \leqslant 2$. Согласно уравнению (11) $\psi$ и удовлетворяет уравнению

$$
\partial_{t}^{2}(\psi u)+2 b \partial_{t}(\psi u)-A(\psi u)-f(t, \psi u)=g_{\psi},
$$

где $g_{\psi}=-f(t, \psi u)+\psi f(t, u)+\psi g+u \psi^{\prime \prime}+2 \partial_{t} u \psi^{\prime}+2 b u \psi^{\prime}$.

Рассмотрим следующую задачу:

$$
\begin{aligned}
& \partial_{t}^{2} \bar{u}+2 b \partial_{t} \bar{u}-A \bar{u}-f(t, \bar{u})=g(t, x), \\
& \left.P_{+} \bar{u}\right|_{t=T}=P_{+}(\psi u)(T),\left.\quad P_{+} \partial_{t} \bar{u}\right|_{t=T}=P_{+} \partial_{t}(\psi u)(T) .
\end{aligned}
$$

Уравнение (17) имеет вид (11). Аналогично работе [1] из теоремы 2 устанавливаются неравенства

$$
\begin{aligned}
\left\|P_{-}(\psi u-\bar{u})\right\|_{\bar{W}(T)} & \leqslant C_{1}\left\|g_{\psi}-g\right\|_{W(T)}, \\
\left\|u_{+}(t)-\bar{u}_{+}(t)\right\|_{\bar{W}(T)} & \leqslant C_{2}\left\|u_{-}(t)-\bar{u}_{-}(t)\right\|_{\bar{W}(T)},
\end{aligned}
$$

где $C_{1}$ и $C_{2}-$ константы, зависяшие от $\|u(\tau)\|$ и $\left\|\partial_{t} u(\tau)\right\|$. Далее, через $C_{i}, i>2$, также обозначаются константы, зависящие от начальных условий функции $u$ в момент времени $\tau$, но не зависящие от $T$.

Очевидно, что $g_{\psi}=g$ при $\tau+1 \leqslant t \leqslant l-1$. Поэтому

$$
\begin{aligned}
\left\|g_{\psi}-g\right\|_{W(T)} \leqslant & C_{3} e^{-(r+b)(T-\tau)} \sup _{\tau \leqslant t \leqslant \tau+1}\left[\|u(t)\|+\left\|\partial_{t} u(t)\right\|+\|g(t)\|+K\right] \\
& +C_{4} e^{(r-b)(T-l)} \sup _{l_{2} \leqslant t \leqslant l_{2}+1}\left[\|u(t)\|+\left\|\partial_{t} u(t)\right\|+\|g(t)\|+K\right] .
\end{aligned}
$$

Из (9), (20) и (18) следует, что при достаточно больших $l$

$$
\left\|P_{-}(\psi u-\bar{u})\right\|_{\bar{W}(T)} \leqslant C_{5} e^{-(r+b)(T-\tau)} .
$$

Так как $\psi u=u$ при $t \in[\tau+1, T]$, то

$$
\left\|P_{-}(u-\bar{u})\right\|_{\bar{W}(T)} \leqslant C_{6} e^{-(r+b)(T-\tau)} .
$$

Из определения пространства $\bar{W}(T)$ и теоремы о следах (см. [2]) следует, что

$$
\begin{aligned}
\|(u-\bar{u})(t)\|_{\Sigma} & \leqslant\|(u-\bar{u})\|_{\bar{W}(T)} e^{(r+b)(T-t)} \\
\left\|\left(\partial_{t} u-\partial_{t} \bar{u}\right)(t)\right\|_{1 / 2} & \leqslant\|(u-\bar{u})\|_{\bar{W}(T)} e^{(r+b)(T-t)}
\end{aligned}
$$

Из (19), (21), (22) получим при $\tau<t<T$

$$
\|u(t)-\bar{u}(t)\| \leqslant C_{7} e^{-(r+b)(t-\tau)}, \quad\left\|\left(\partial_{t} u-\partial_{t} \bar{u}\right)(t)\right\|_{1 / 2} \leqslant C_{7} e^{-(r+b)(t-\tau)} .
$$

В частности, $\|u(\tau)-\bar{u}(\tau)\|_{\Sigma} \leqslant C_{7}$ и $\left\|\partial_{t} u(\tau)-\partial_{t} \bar{u}(\tau)\right\|_{1 / 2} \leqslant C_{7}$. 
Возьмем произвольную последовательность $\left\{T_{j}\right\}_{j=1}^{\infty}, T_{1}=T, T_{j} \rightarrow \infty$. Для каждого $T_{j}$ указанным выше способом фиксируем функцию $\bar{u}_{j}$. Как было установлено,

$$
\left\|u(t)-\bar{u}_{j}(t)\right\|_{\Sigma} \leqslant C_{7} e^{-(r+b)(t-\tau)} \quad \text { и } \quad \bar{u}_{j} \in V_{2}(t, t+1)
$$

при $t \in\left[\tau, T_{j}\right]$.

Множество точек $\left(P_{+} \bar{u}_{j}(\tau), P_{+} \partial_{t} \bar{u}_{j}(\tau)\right)$ лежит в шаре конечномерного пространства $E_{+} \times E_{+}$. Обозначим через $\left(u_{\infty}, u_{\infty}^{\prime}\right)$ произвольную предельную точку этого множества. Пусть $v \in \bar{W}(\tau)$ - решение уравнения (11) при начальном условии $\left.P_{+} v\right|_{t=\tau}=u_{\infty}$, $\left.P_{+} \partial_{t} v\right|_{t=\tau}=u_{\infty}^{\prime}$. Функция $v$ непрерьвно зависит от начальных условий. Следовательно, из (23) имеем, что

$$
\|u(t)-v(t)\|_{\Sigma} \leqslant C_{8} e^{-(r+b)(t-\tau)}, \quad t>\tau .
$$

Теорема доказана.

ДокАЗАТЕЛЬСТво ТЕОРЕмЫ 1 . Пункт 1) теоремы 1 следует из теорем 2 и 3 . Докажем пункт 2). Пусть $U(t), t>\tau,-$ произвольное решение задачи $(1),(8)$. Согласно (9) существует такое число $T>0$, что $\|U(t)\| \leqslant \frac{2}{3} \rho$ при $t \geqslant T$. Обозначим $u(t)=U(t-\tau+T)$. Тогда функция $u(t)$ удовлетворяет уравнению (11) при начальном условии $\left.u\right|_{t=\tau}=U(T)$. Значит, по теореме 3 сушествует решение $v(t) \in M$ уравнения (11), для которого выполнено соотношение (16). Так как $u(t) \in \Lambda^{a}(\tau+T)$ экспоненциально притягивается $\mathrm{k}$ функции $v$, то $v(t) \in \Lambda^{a}(\tau+T)$ и $\|v(t)\|_{\Sigma} \leqslant \rho$ для всех $t$, больших некоторого $T_{0}>T$. Поэтому функция $v(t)$ является решением уравнения (1) при $t>\tau+T_{0}$. Тогда неравенство (14) следует из (16). Теорема 1 доказана.

ЗАмечаниЕ. Теорема 1 остается справедливой для любой области $\omega \Subset \mathbb{R}^{n}$ с гладкой границей и линейного самосопряженного оператора $A$, удовлетворяющих спектральному условию (13).

Московский государственный университет

Поступило

им. М. В. Ломоносова

03.12 .96

\section{СПИСОК ЦИТИРОВАННОЙ ЛИТЕРАТУРЫ}

1. Babin A. V. Inertial manifolds for travelling-wave solutions of reaction diffusion systems // Comm. Pure Appl. Math. 1996. 2. Лионс ЖК.-Л., Мадженес Э. Неоднородные граничные задачи и их приложения. М.: Мир, 1971. 3. Бабин А. В. // Изв. РАН. Сер. матем. 1994. Т. 58. №2. С. 3-18. 4. Вишик М. И., Зелик С. В. // Матем. сб. 1996. Т. 187. № 12. С. 21-56. 